\title{
Airlines provide too little information for allergy sufferers!
}

\author{
Jürgen Seidenberg · Geeske Stelljes · Lars Lange · Katharina Blumchen · Ernst Rietschel
}

Received: 27 May 2020 / Accepted: 5 August 2020 / Published online: 20 November 2020

(c) The Author(s) 2020

\begin{abstract}
Summary
Introduction Allergic reactions are reported to account for approximately $2-4 \%$ of all medical emergencies on commercial airline flights. In 2016, the International Air Transport Association (IATA) published recommendations on risk prevention in severe allergies.

Methods Using a written questionnaire and an internet search, an investigation was conducted on the extent to which airlines operating in Germany have implemented the IATA recommendations and, e.g., offer peanut/nut allergy sufferers appropriate measures. Results Only 14 of the 104 airlines contacted responded to the written survey. Of 115 airlines, 72 provided information for allergy sufferers on their
\end{abstract}

Prof. Dr. J. Seidenberg $(\bowtie) \cdot$ G. Stelljes

University Department of Pediatrics and

Adolescent Medicine, Klinikum Oldenburg,

Carl von Ossietzky University Oldenburg,

Rahel-Straus-Straße 10, 26133 Oldenburg, Germany

seidenberg.juergen@googlemail.com

Prof. Dr. J. Seidenberg · Dr. L. Lange ·

Priv.-Doz. Dr. K. Blumchen · Priv.-Doz. Dr. E. Rietschel

Anaphylaxis Scientific Working Group, Society for Pediatric

Allergy and Environmental Medicine, Aachen, Germany

Dr. L. Lange

Department of Pediatrics, St. Marien-Hospital, Bonn,

Germany

Priv.-Doz. Dr. K. Blumchen

Department of Pediatrics and Adolescent Medicine, Pneumology, Allergology, and Cystic Fibrosis, University Hospital Frankfurt, Goethe University, Frankfurt/Main, Germany

Priv.-Doz. Dr. E. Rietschel

Department of Pediatrics and Adolescent Medicine, Medical Faculty, University Hospital Cologne, Cologne, Germany homepage, but mostly in insufficient detail. No results were found for the search term "allergy" (or "Allergie") on the websites of 43 airlines. The information on the individual airlines has been summarized in table form.

Discussion The information offered by many airlines for passengers with allergies is insufficient. To offer greater guidance, updated information has been formulated in German and English, and its use is recommended.

Keywords Peanut allergy · Nut allergy · Anaphylaxis . Allergy · Air travel

\section{Introduction}

Allergic reactions are reported to account for approximately $2-4 \%$ of all medical emergencies on commercial airline flights [1]. This number is likely to be higher, since acute asthma attacks are documented as a respiratory problem and not necessarily as a symptom of a severe allergic reaction [2]. In addition, two thirds of passengers do not inform flight crews of their allergic reaction during the flight [3]. When asked, 9\% of peanut allergy sufferers reported experiencing an in-flight allergic reaction [4].

Severe allergic reactions on commercial flights are primarily caused by foods (peanuts, tree nuts, or seafood) and medications [1]. Anaphylactic shock is the most extreme form of allergic reaction. Within minutes, hives, swelling in the face and neck region, shortness of breath due to asthma, vomiting, and circulatory failure can develop, with fatal consequences. For some allergy sufferers, the thought of experiencing an allergic reaction of this kind while on a cramped aircraft without medical assistance is extremely frightening. This can also lead to disproportionate anxiety and challenges, particularly if no 
Table 1 Questionnaire sent to the airlines

Questionnaire on how airlines deal with the risk of anaphylaxis in peanut/nut allergy

\section{Anaphylaxis prevention}

Do you have guidelines for passengers with allergies?

$\square$ Yes

If so, are these guidelines visible on your website?

$\square$ Yes

$\square$ No

Do you advise passengers that, if they have severe allergies, they should always carry one or more adrenaline pens on them, as well as any other medications they may require?

$\square$ Yes

Do you advise passengers that allergy sufferers requiring care (e.g., minors, disabled persons) are not permitted to travel alone?

$\square$ Yes

Do you offer allergic passengers the opportunity to board the aircraft before other passengers and clean the seats in their vicinity themselves in order to remove allergen components?

$\square$ Yes, they are permitted to board the aircraft before other passengers and clean

$\square$ Yes, they are permitted to clean, but not to board the aircraft before other passengers

$\square$ No, they are not permitted to clean other seats

Do you advise allergic passengers that the airline is not able to guarantee an allergen-free flight?
$\square$ Yes
$\square$ No

Do you offer allergic passengers an appropriate buffer area in which special measures will be taken to minimize their risk of allergic reactions?

$\square$ Yes

$\square$ No

If yes:

How many rows of seats does the buffer area cover?

$\square$ Only the row in which the allergy sufferer is seated

$\square$ One row behind and in front

$\square$ Additional rows of seats behind and in front, №.:

Are allergy sufferers given the opportunity, e.g., to clean the seats in the

buffer area of residual allergens using wet wipes?

$\square$ Yes $\quad \square$ No

Are all passengers in the buffer area requested not to consume peanuts/ nuts?
$\square$ Yes
$\square$ No

In addition, are all passengers requested not to consume peanuts/nuts?

$\square$ Yes

$\square$ No

If no (no buffer area):

Are all passengers requested not to consume peanuts/nuts?

$\square$ Yes, this is requested of all passengers

$\square$ No, this is not requested of passengers

Are foods that are labeled as containing the relevant allergen (in the list of ingredients) excluded from sale or distribution to passengers?

$\square$ Yes, for all passengers

$\square$ Yes, but only for passengers in the buffer area (if offered)

$\square$ No

Are foods that are labeled only as "may contain traces of peanuts/nuts" also excluded from sale or distribution to passengers?

$\square$ Yes, for all passengers

$\square$ Yes, but only for passengers in the buffer area (if offered)

$\square$ No

Does the allergy sufferer have the option to order a special (peanut-/nut-free) meal for themselves or the person(s) accompanying them?

$\square$ Yes

$\square$ No

If yes:

$\square$ With prior notification at least $24 \mathrm{~h}$ before boarding

$\square$ With prior notification at least $48 \mathrm{~h}$ before boarding

$\square$ With prior notification at least $72 \mathrm{~h}$ before boarding

$\square$ With earlier prior notification

$\square$ Without prior notification 
Table 1 (Continued)

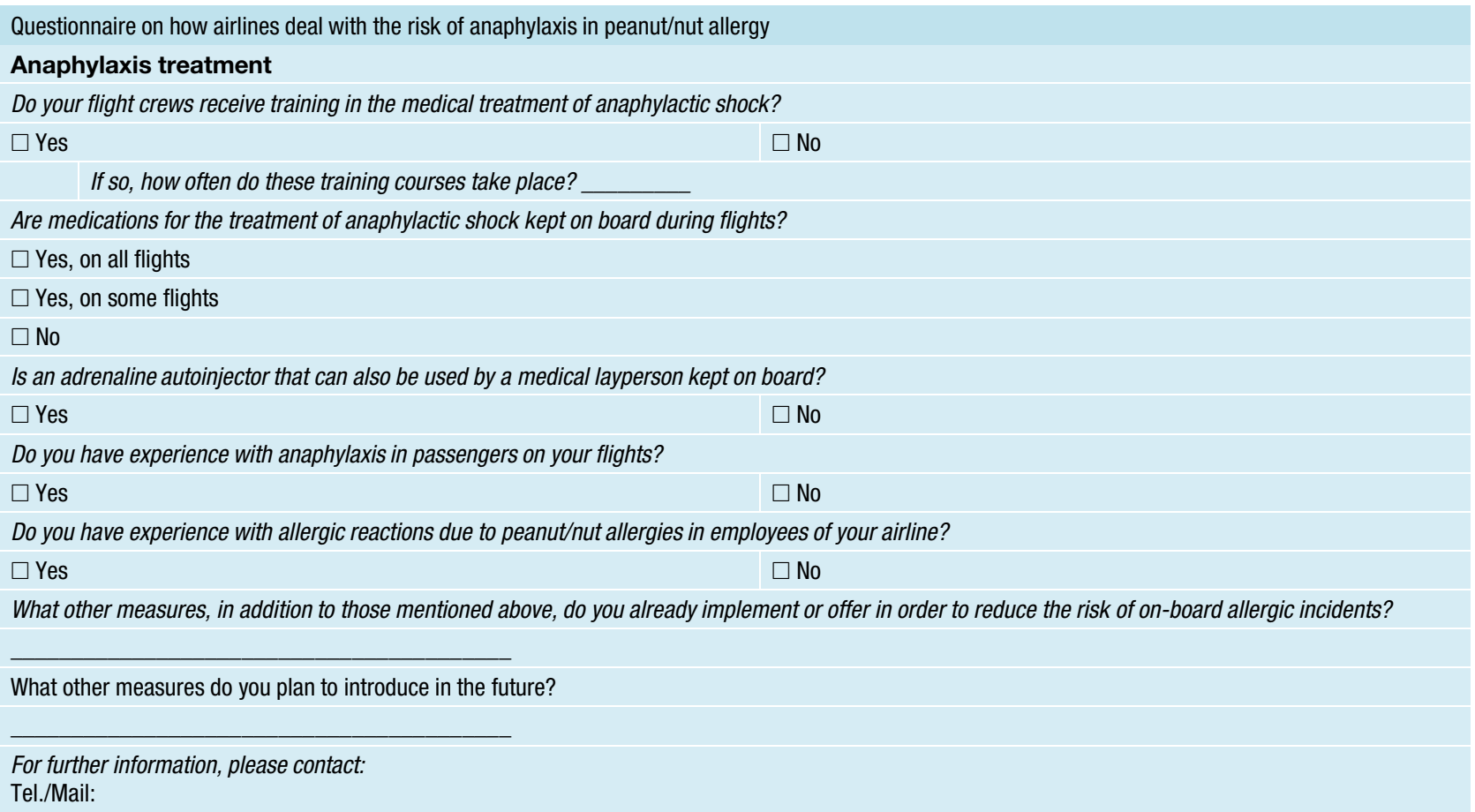

training and education on the causes and treatment of anaphylaxis have been provided [5].

People not suffering from allergies, on the other hand, often display no understanding for the potential risk to life posed by even small quantities of certain foods. Still today, individual case reports [6-8] attest to the unreasonable behavior of fellow travelers and considerable uncertainty among flight crews on how to deal with allergy sufferers due to a lack of appropriate information and structured guidelines from the airline. However, the in-flight risk of anaphylaxis could be significantly reduced by just a few simple measures taken by the patient themselves as well as the airline [9]. In 2016, the International Air Transport Association (IATA) published a communication, aimed at both allergy sufferers and airlines, containing recommendations on appropriate measures [10]. Air Canada and a number of other airlines have meanwhile largely adopted these guidelines [11].

To obtain information on the extent to which airlines operating in Germany have implemented the IATA recommendations and are now offering appropriate measures for passengers with severe allergies (e.g., peanut/nut allergy), the authors made a telephone inquiry to the German Federal Aviation Office (Luftfahrt-Bundesamt, LBA). They learned that there is no Germany-wide regulation and that those affected need to address their queries directly to the relevant airline. Travel agents and tour operators also need to contact the respective airline, or search through its website, in each individual case. The guidelines vary considerably here. Therefore, we conducted a written survey on this topic among the airlines operating in Germany.

\section{Methods}

In spring 2019, questionnaires (Table 1) were sent to 104 airlines operating in Germany. The questions could be answered quickly and simply by ticking a box. Airlines that failed to reply received two reminders within 3 months, with the questionnaire enclosed once again, both in German and English.

In a second step, the authors searched the airlines' websites for information aimed at allergy sufferers. One cannot rule out the possibility that hidden information was overlooked and that not all airlines operating in Germany were recorded.

\section{Results}

The detailed results of the survey and online research are presented in Table 2. Data that came directly from the airlines are shown in normal type, while the information found by the authors is presented in italics. Table 2 provides information on 115 airlines. For 11 airlines, it became apparent only later that they also operate in Germany. These airlines did not receive questionnaires due to time constraints. As such, 104 airlines were contacted in writing up to three times with the questionnaire. Only four airlines returned their completed questionnaires. A total of 10 others responded in writing, albeit incompletely. The remaining airlines did not respond, despite two reminders. 


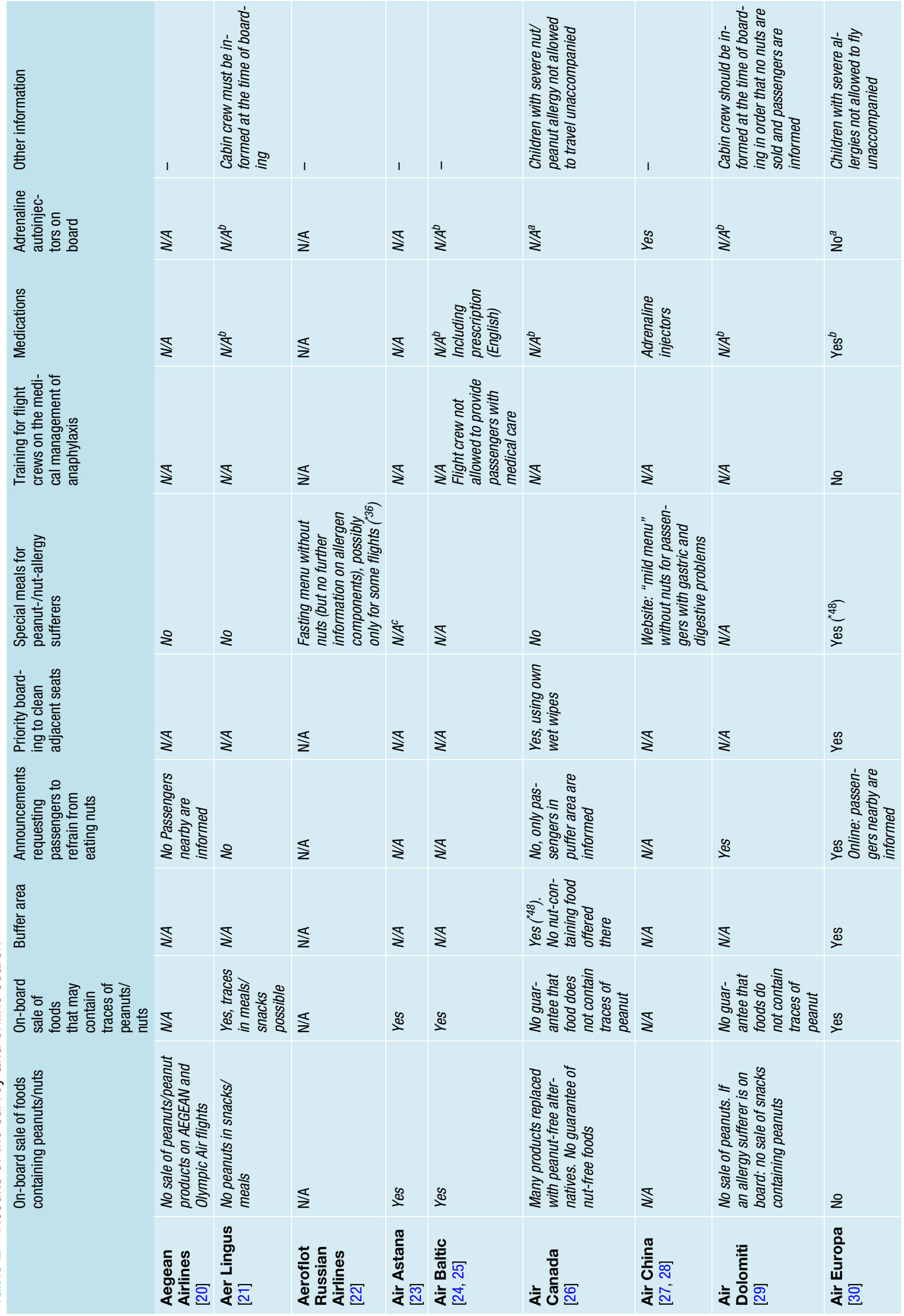




\section{original article}
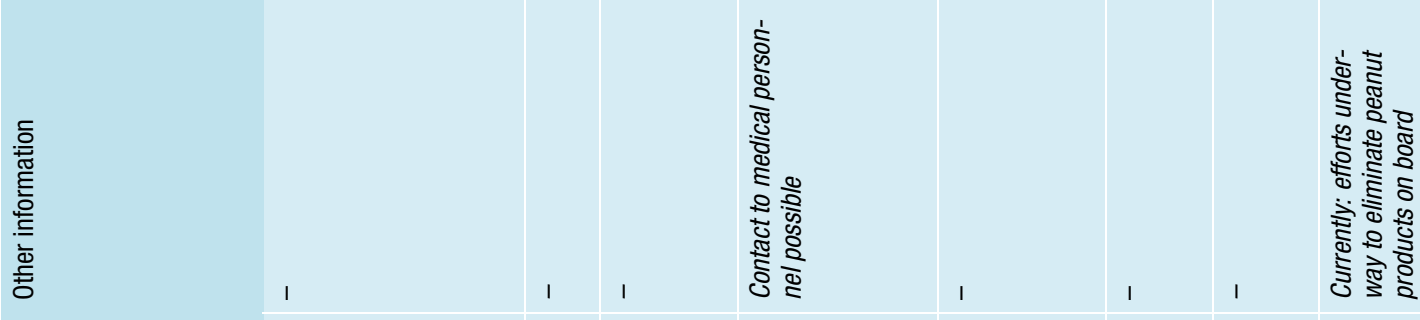

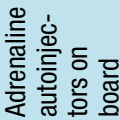

$\stackrel{n}{2}$

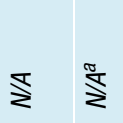

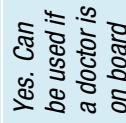

$\stackrel{\pi}{3}$

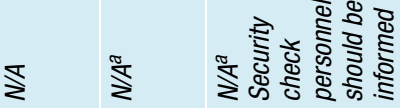

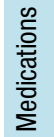

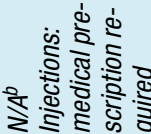

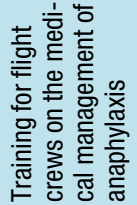

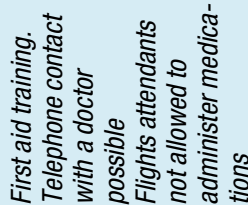

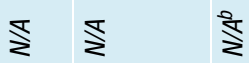

$\stackrel{1}{2}$

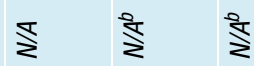

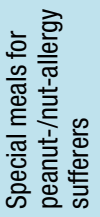

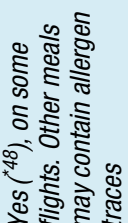

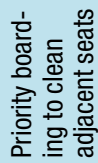

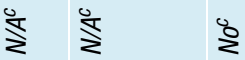

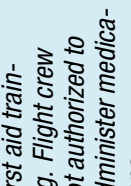

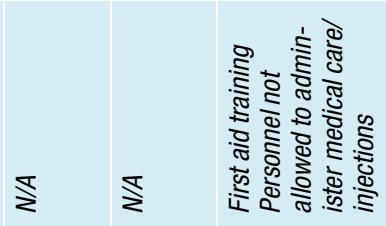

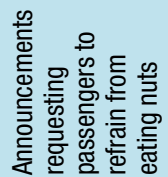

$\stackrel{\pi}{e}$

$\stackrel{\pi}{2}$
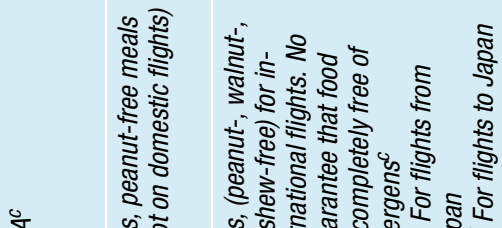

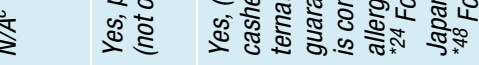

丞

$\stackrel{5}{2}$

造

$\stackrel{\pi}{2}$

$\stackrel{\pi}{\Sigma}$

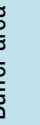

$\stackrel{1}{2}$

$\pi$

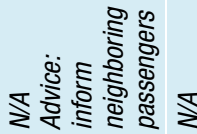

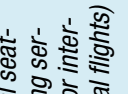

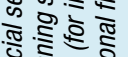

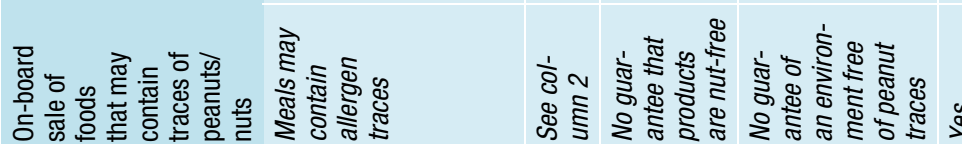

र

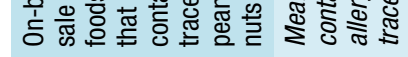

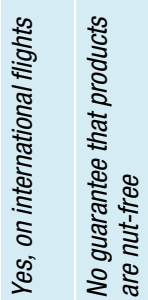

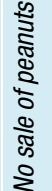

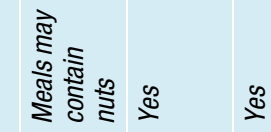
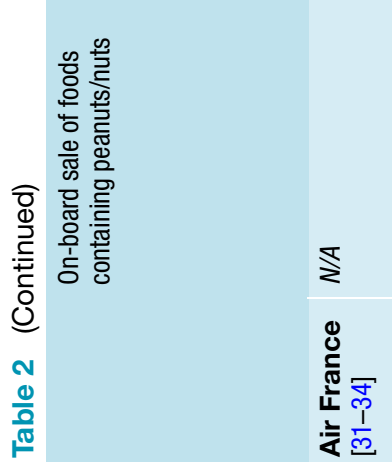

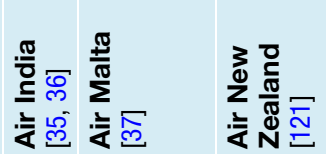

\&

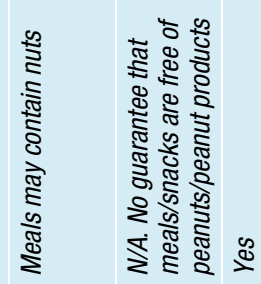

先

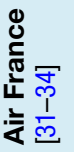

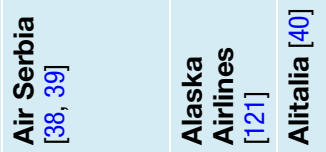

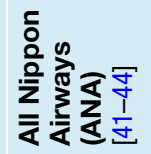




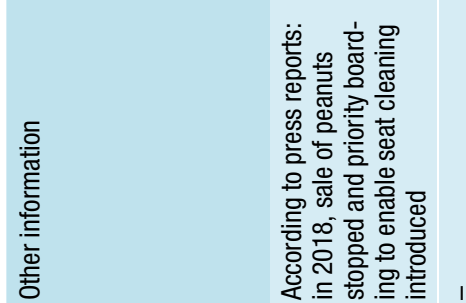

IIs

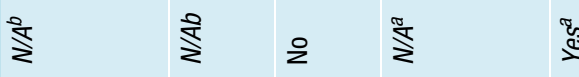

$\stackrel{\pi}{2}$

$\stackrel{T}{2}$

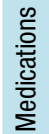

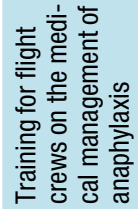

$\stackrel{n}{2}$

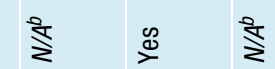

$\stackrel{8}{2}$

$\stackrel{\Gamma}{\Sigma}$

$\stackrel{\pi}{2}$

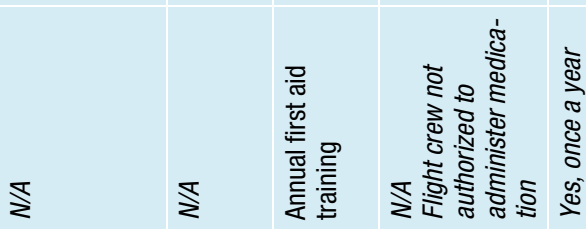

$\stackrel{\pi}{\Sigma}$

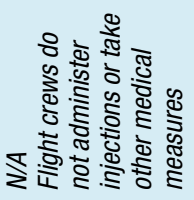

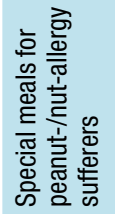

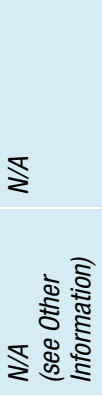

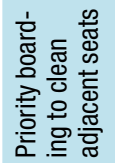

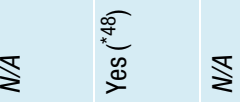

$\gtrless$

$\stackrel{\Gamma}{2}$

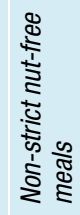

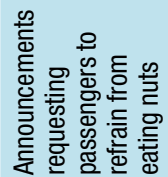

『্

\& $2 \quad$ \&

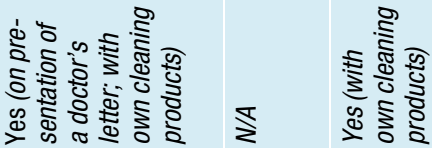

$\stackrel{\pi}{2} \stackrel{\pi}{2}$

$\stackrel{\pi}{2}$

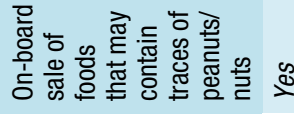

\& $\quad \stackrel{\pi}{2}$

$\gtrless$

$\gtrless$

$\$$

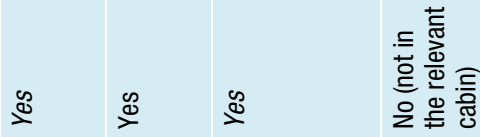

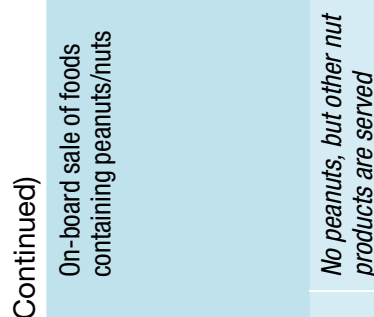

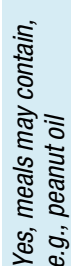

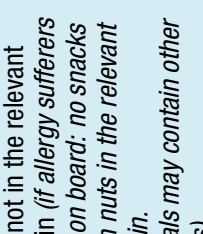

\&

$\stackrel{5}{2}$

$\frac{\sqrt{\pi}}{\frac{0}{0}}$

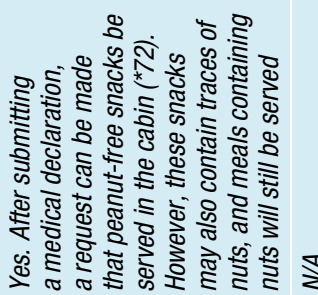




\section{original article}

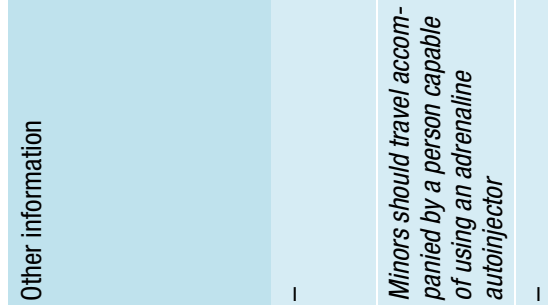

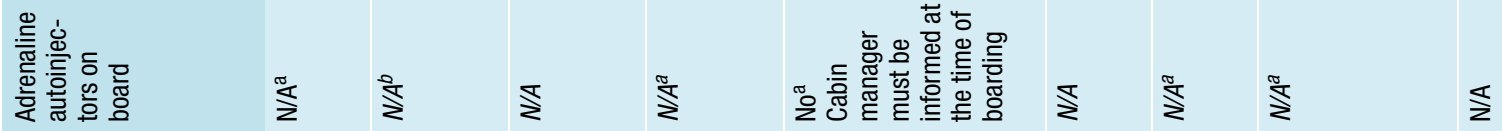

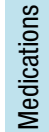

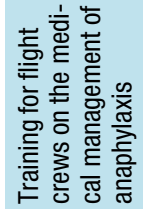

2 $\quad$ i

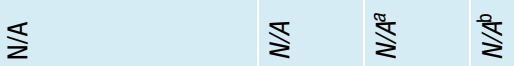

$\stackrel{5}{2}$

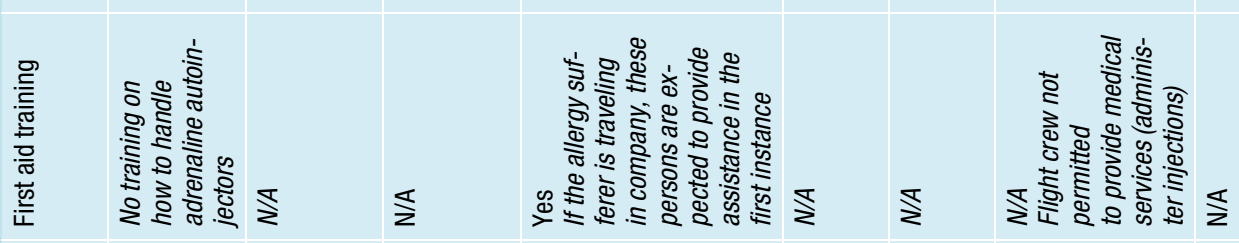

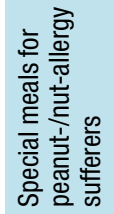

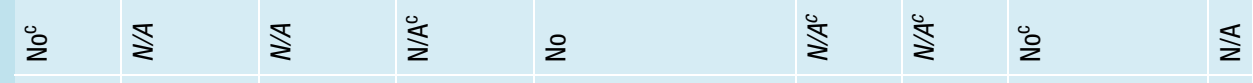

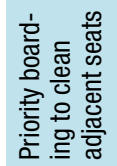

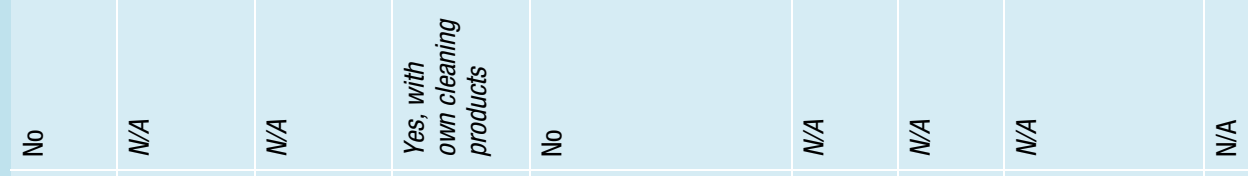

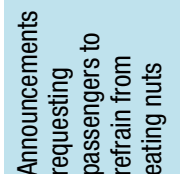

$\gtrless \quad \gtrless$

$\stackrel{5}{z}$

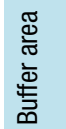

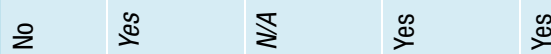

22

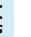

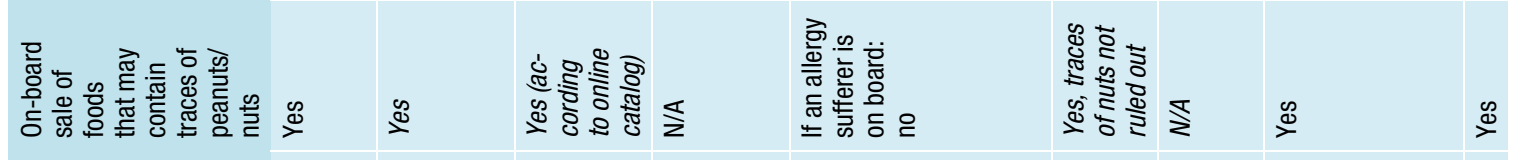

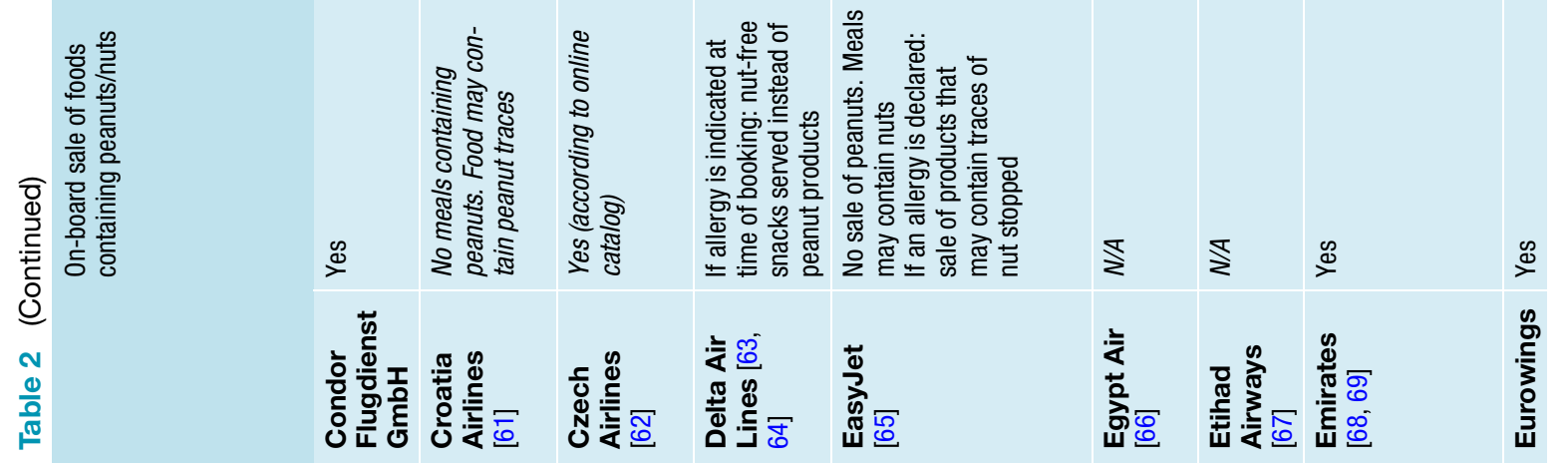




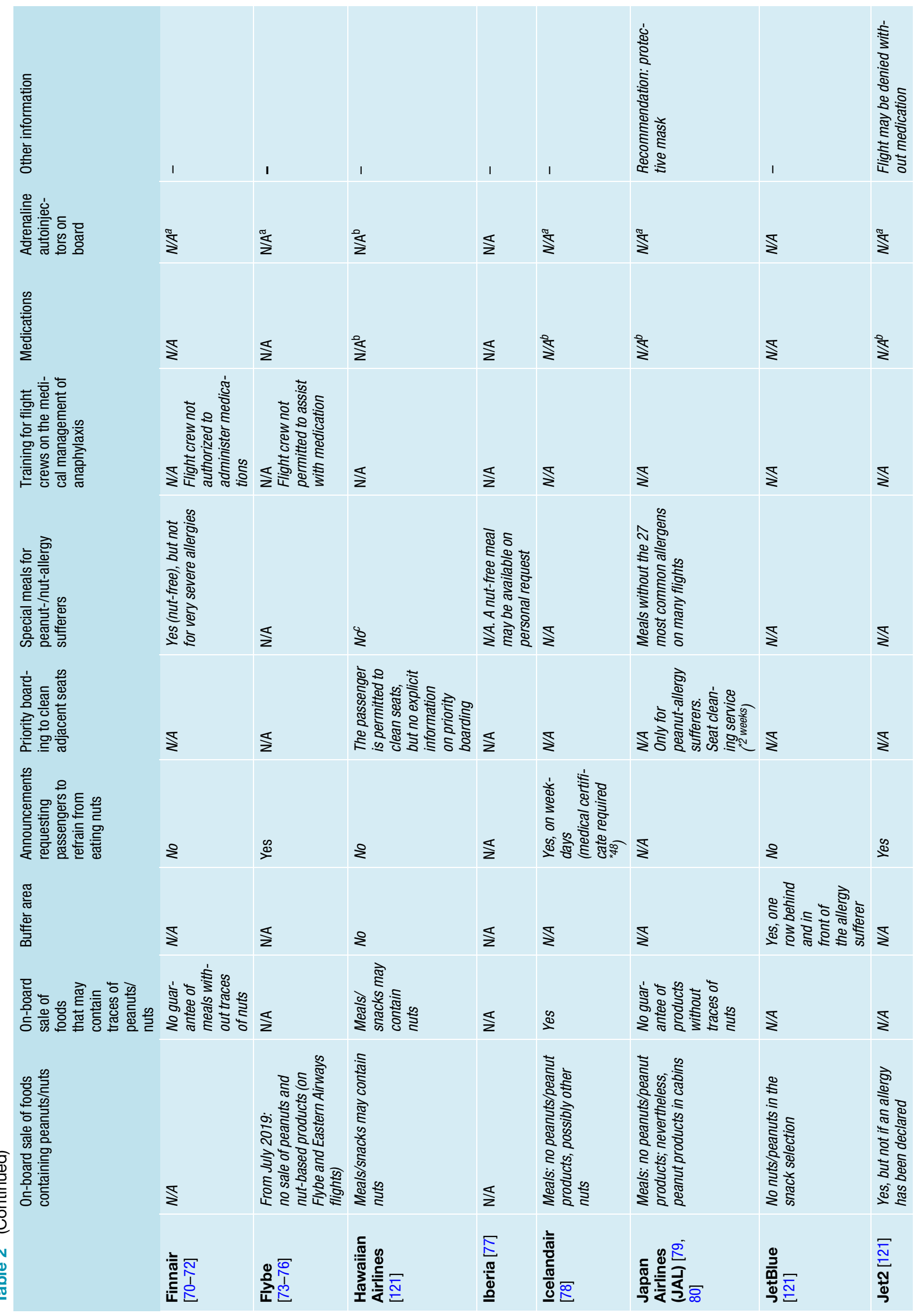




\section{original article}

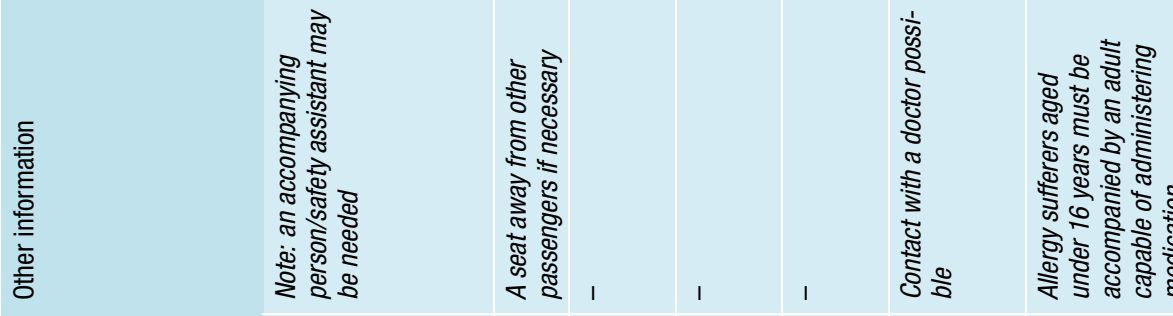

Ines

$\stackrel{\pi}{\Sigma}$

i $\frac{\pi}{2} \quad \frac{\pi}{2}$

$\stackrel{n}{2}$

$\stackrel{\pi}{\Sigma}$

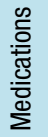

$\stackrel{2}{2}$

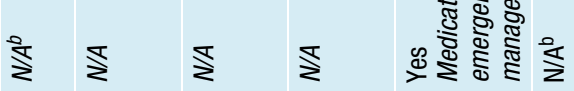

$\stackrel{2}{2}$

$\stackrel{n}{2}$

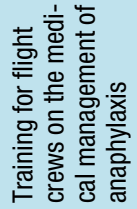

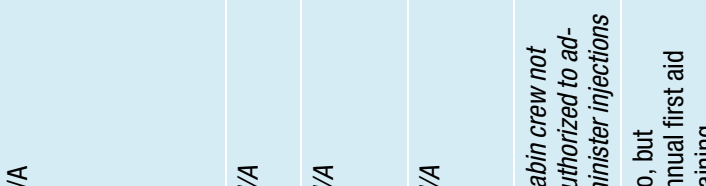

$\stackrel{s}{\Sigma}$

₹

$\stackrel{\pi}{2}$

$\stackrel{5}{2}$
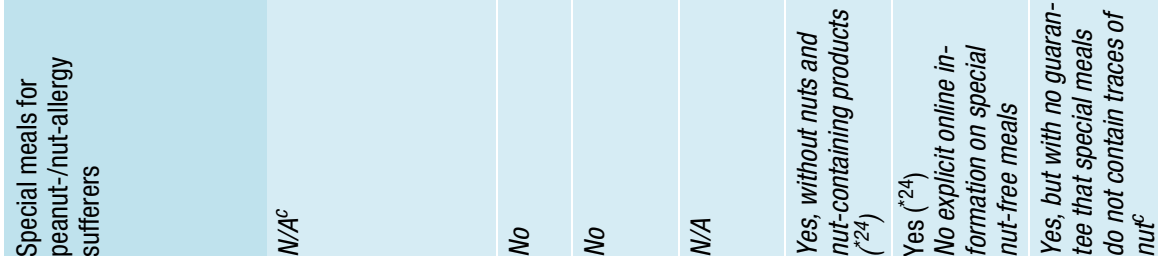

$\stackrel{i}{2}$

$\gtrless \&$

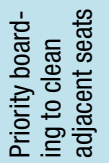

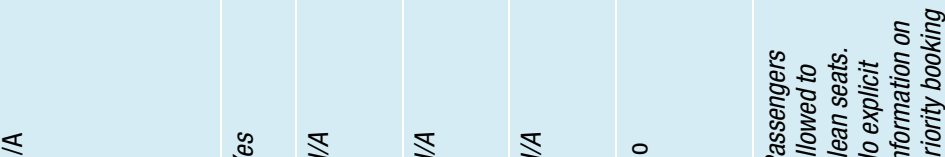

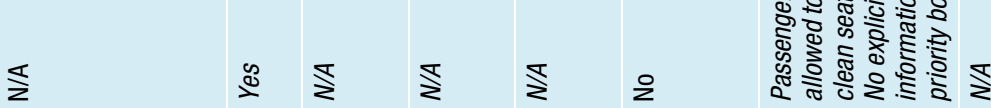

$\stackrel{\varpi}{\Sigma}$

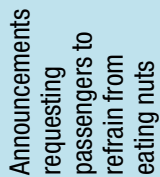

$\stackrel{x}{2}$

$\stackrel{\pi}{2}$

$\stackrel{T}{2}$

$\gtrless$

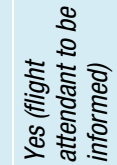

总

$\stackrel{s}{z}$

$\stackrel{\pi}{\pi} \stackrel{x}{\pi}$

$\$$

$\$$

매해

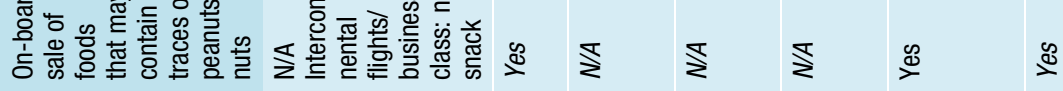

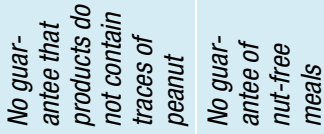
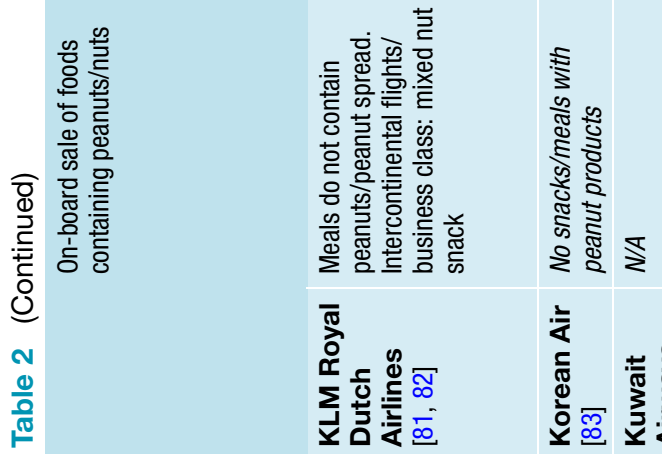

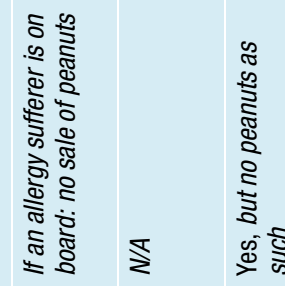
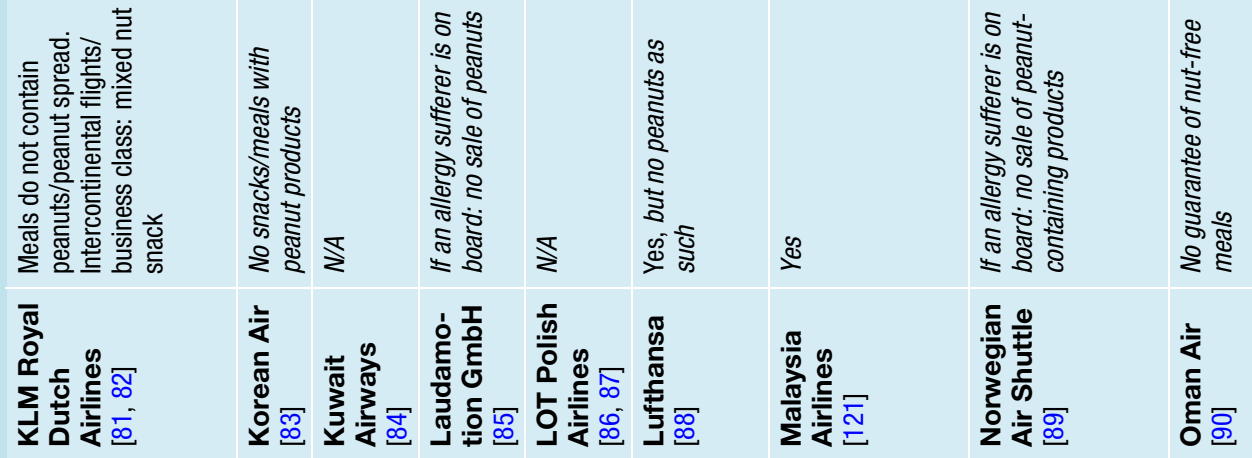


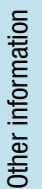

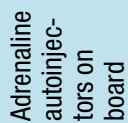

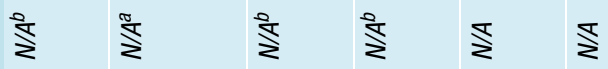

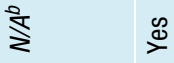

$\stackrel{n}{2}$

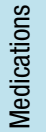

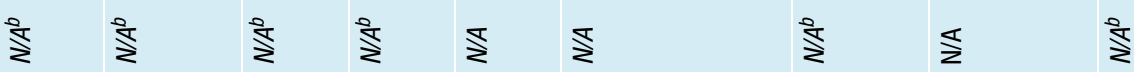

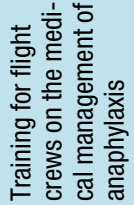

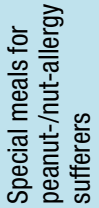

$\stackrel{\pi}{i} \quad \frac{\pi}{2} \quad \frac{\pi}{2}$

$\stackrel{\pi}{2} \quad \stackrel{2}{z}$

$\stackrel{\pi}{2}$

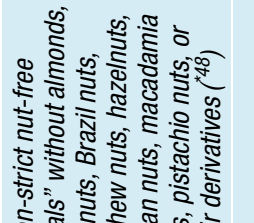

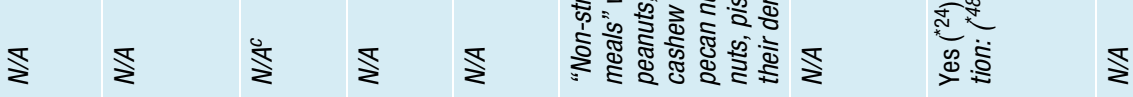

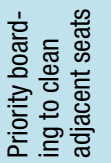

$\frac{\pi}{\pi} \quad \frac{1}{\pi} \quad \frac{1}{\pi}$

$\stackrel{\pi}{2}$

$\stackrel{\pi}{2}$

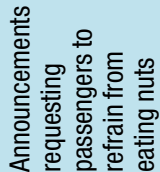

丞

$\frac{1}{\pi} \sqrt{\pi} \frac{\pi}{2}$

$\stackrel{2}{2}$

$\stackrel{\pi}{\Sigma}$

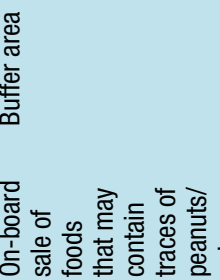

交

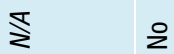

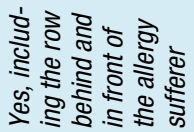

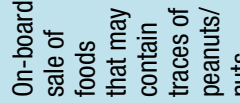
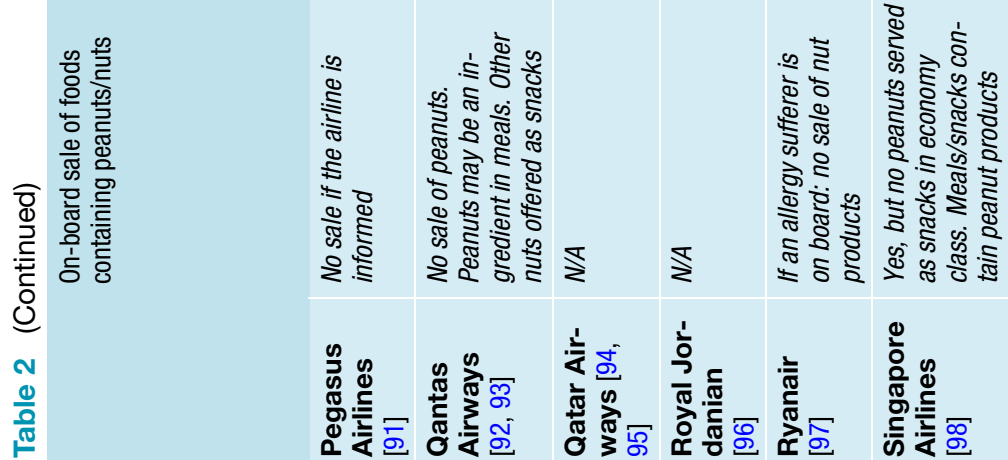

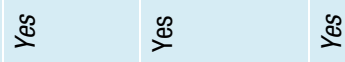

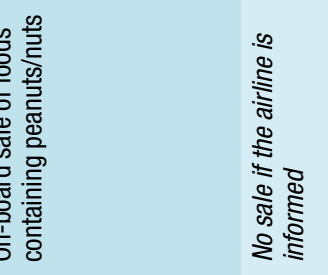

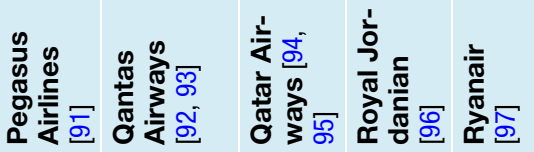
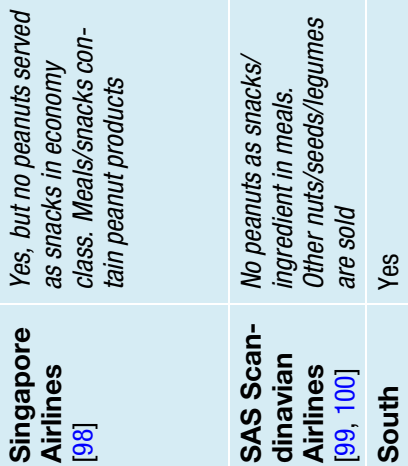

$\stackrel{8}{2}$

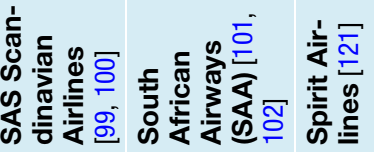




\section{original article}

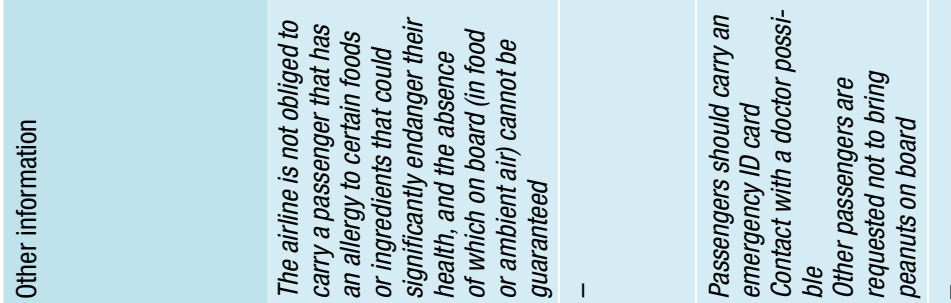

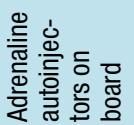

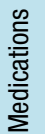

$\stackrel{\pi}{\Sigma}$

$\stackrel{\pi}{2}$

$2 \stackrel{\pi}{2}$

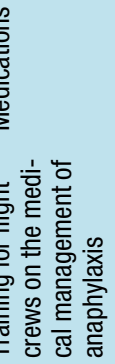

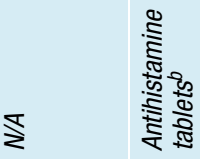

$2 \stackrel{\pi}{2} \sum^{\pi}$

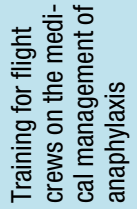

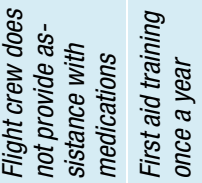

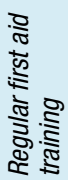

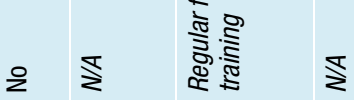

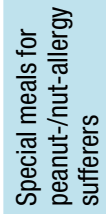

$\stackrel{5}{2}$

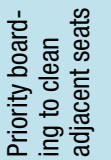

$\stackrel{5}{2}$

\&

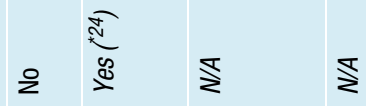

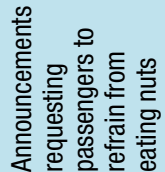

$\stackrel{5}{2}$

$\stackrel{\pi}{\Sigma}$

$\frac{\pi}{2} \stackrel{\pi}{2}$

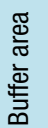

$\stackrel{5}{2}$

$\stackrel{x}{z}$

$2 \lessgtr$

$\stackrel{\Gamma}{2}$

$\stackrel{\pi}{2}$

$2 \stackrel{\pi}{2}$

$\stackrel{\pi}{2}$

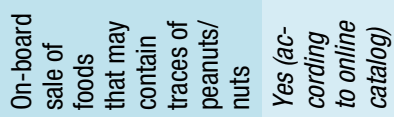

के ㅎํㅎำ के

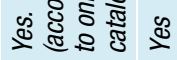

$\stackrel{\infty}{\longleftarrow}$
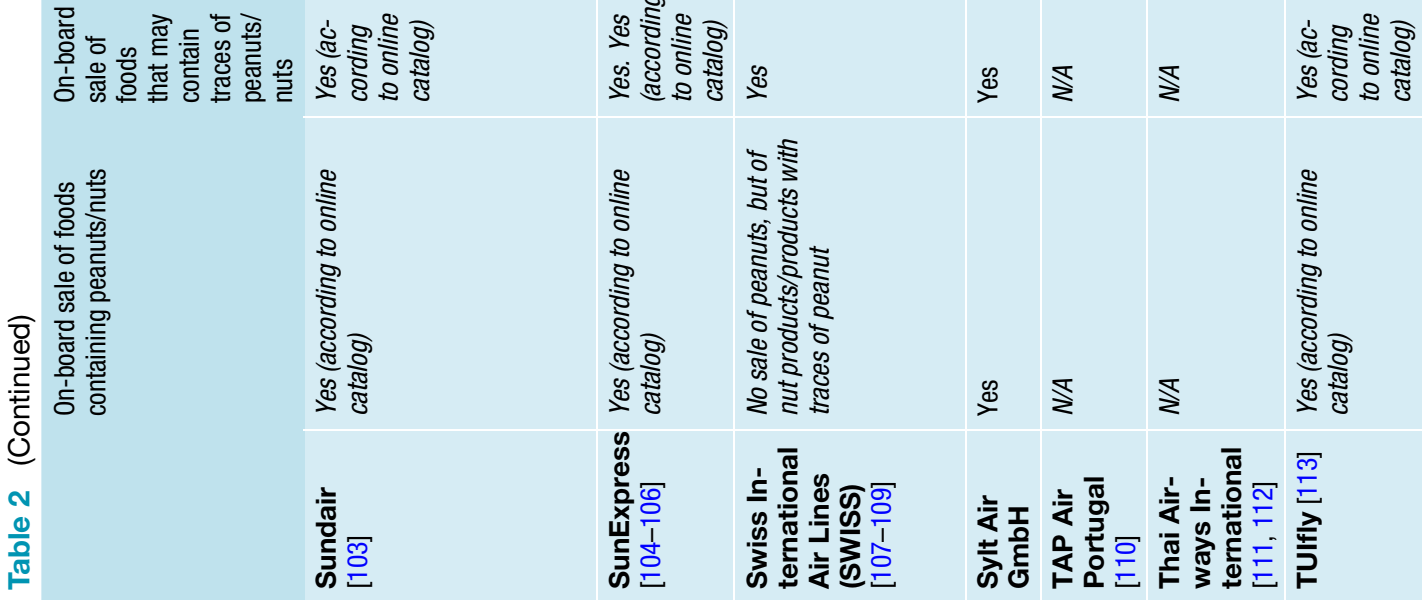

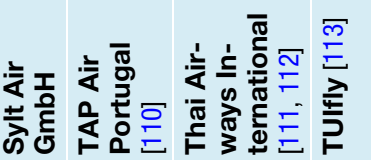




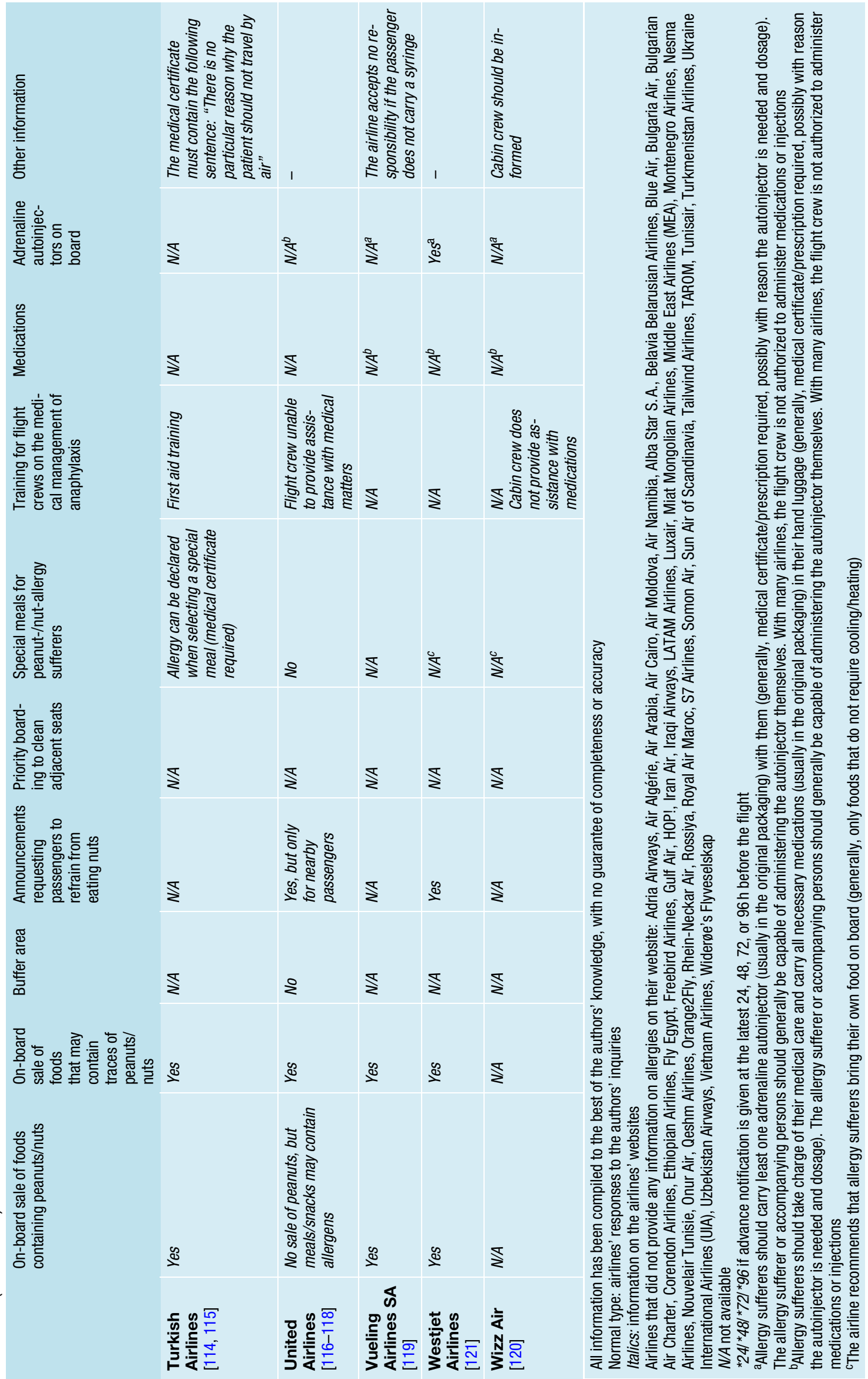


Considerable variance was seen on the airlines' websites. In all, 72/115 airlines provided information for allergy sufferers on their homepage, but mostly in insufficient detail. For 43 airlines, not even the search term "allergy" (or "Allergie") produced any results on their websites.

Almost all airlines that make reference to allergies emphasize that they are unable to guarantee an allergen-free environment or a completely "nut-free flight" and therefore do not accept responsibility for in-flight allergic reactions. Nevertheless, 26 airlines do not serve snacks containing peanuts or offer to refrain from doing so for passengers with allergies. A total of 31 airlines continue to sell snacks containing peanuts or nuts. No data is available for the remaining airlines. Four airlines said that they provided a buffer area where necessary, while 12 do not. There are no data for the other airlines. Six airlines reported operating priority boarding for allergy sufferers, enabling these passengers to wipe down their seating area with their own wet wipes. Interestingly, two Japanese airlines offer their own special seat-cleaning service on request. Most airlines explicitly require affected passengers to inform them in advance of any allergies. However, how and when this should be done varies widely. Many airlines require a medical clearance certificate or a preprinted form to be filled out. Sometimes, these certificates are only accepted if preformulated sentences are used and time limits are observed. Medications carried in hand luggage need to be declared and approved in some cases, and information is also requested when boarding. Documents often also need to be provided in English.

A total of 15 airlines state that they offer varying degrees of strictly allergen-free meals for peanut/nut allergy sufferers. However, it is often not clear from this internet-based information whether meals may also contain traces of allergens. If an airline does offer special nut-free meals, these need to be ordered between 24 and $48 \mathrm{~h}$ before the flight. Cabin announcements are only allowed with the cabin manager's authorization. Only five airlines carry adrenaline autoinjectors.

In summary, there are major differences between the airlines in terms of their approach to allergy sufferers. On a positive note, a small number of airlines are now addressing this problem in a targeted manner and have introduced risk-reduction measures. Some airlines make reference to the IATA communication. However, several airlines that are IATA members provide no information for allergy sufferers on their websites.

\section{Discussion}

The results of this survey clearly show that more information and action is needed in order to minimize the risk of life-threatening in-flight anaphylaxis for severe allergy sufferers. This is a task not only for the many airlines and their staff, but also for passen- gers with allergies, who should likewise be encouraged to make appropriate preparations to ensure low-risk travel. The best way to ensure that these preparations are made is if the airline requests them prior to the flight. To this end, all airlines should make relevant information available on their websites.

However, the IATA recommendations issued in 2016 need to be updated. For example, the European Medicines Agency (EMA) has recommended since 2015 that patients carry two adrenaline autoinjectors [12]. One rationale behind this is that the effect of adrenaline can significantly diminish within as little as $1 \mathrm{~h}$, and a persistent anaphylactic reaction requires a further adrenaline dose to be administered. In addition, there have been recent reports of autoinjector malfunction, hence, the recommendation to carry two autoinjectors.

Moreover, several studies show that the risk of anaphylaxis due to inhalation of an allergen-containing aerosol, e.g., when opening a pack of nuts at some distance, is extremely low, thereby calling into question the need for all passengers on an aircraft to avoid peanuts outside a small buffer area [13]. However, up to 25-fold higher amounts of peanut protein were detected on aircraft tray tables when peanuts were served as a snack [14]. Another investigation detected peanut particles in aircraft air conditioning systems [15]. Therefore, dispensing with peanut-/nut-containing snacks on commercial flights remains desirable when allergy sufferers are traveling; however, decontamination measures are taking the foreground, e.g., priority boarding for allergy sufferers to enable them to clean the seat upholstery, armrests, and table surfaces in the row with their booked seats using their own wet wipes. Of apparent importance is the information that soap-based commercial wipes are better for peanut allergen removal than alcohol-based disinfection [16]. In the particular context of air travel, the allergic patient needs the acceptance, cooperation, and support of the airline and its flight attendants.

In coordination with the Anaphylaxis Scientific Working Group of the Society for Pediatric Allergy and Environmental Medicine GPA e.V., the authors recommend that all airlines operating in Germany provide readily accessible information for allergy sufferers on their websites or provide information upon request. Flight and travel agencies should inform passengers about this in good time. In order to make a constructive contribution in this regard, the authors have updated the IATA recommendations and made them available in English (see below) and in German (see German version: Allergo Journal 8/2020, www. springermedizin.de/allergo-journal). Not all of the following recommendations have been validated by extensive studies and, thus, merely represent the best possible evidence at the present time.

One element of the IATA recommendations that has not been adopted is the advice on aircraft emer- 
gency kits, since flight crew are not authorized to administer adrenaline in the form of an injectable solution. Although supplementing aircraft kits with adrenaline autoinjectors is evaluated positively [17], these are generally not available and the personnel are not trained in their use. Thus, the authors deem it all the more important to make passengers aware that they themselves need to ensure that they have sufficient emergency medication and are also able to use it accordingly. Children up to the age of 12-14 years need to be accompanied by a trained person [18]. It is also absolutely essential to provide information on the type and severity of the allergy in good time prior to booking a flight. The European Union air passenger rights stipulate that a passenger can be denied boarding if the airline has not been informed in advance of any severe allergies [19]. The following is the authors' recommendation for an information sheet for passengers:

\section{Information for passengers with severe allergies}

To avoid in-flight medical emergencies, passengers are advised to fully comply with the following instructions.

\section{Before travel}

1. You should inform your physician of your intention to travel; he/she should check that your emergency kit is complete and has sufficient dose(s). An emergency kit consists of two adrenaline autoinjectors, one second-generation histamine- $\mathrm{H}_{1}$ receptor antagonist, a systemic glucocorticoid, and, for asthmatics, also an emergency asthma spray. None of these medications should reach their expiry date before the return journey. Your physician needs to issue you with an emergency plan with instructions on allergy diagnosis, dose and route of administration of medications, as well as the need to take these on board; for travelling abroad, this plan should also be in English. Your physician should check whether you (as well as accompanying persons in the case of allergic children) are currently able to use the adrenaline autoinjector. Allergic children should never travel unaccompanied by a trained adult unless they have reached the age of 12-14 years and have demonstrated their ability to self-administer emergency treatment.

2. Taking out travel cancellation and travel interruption insurance, as well as travel health insurance, is recommended.

3. You must inform the airline of the risk of a severe allergic reaction in good time before booking. You should express the wish that, in order to reduce your risk on the flight:

- For food allergies: you be granted priority boarding in order to clean seat upholstery, arm rests, and table surfaces in your row of seats with your own wipes (soap-based cleansing wipes are better for removing, e.g., residual nut than alcoholbased disinfectant wipes).

- For peanut/nut allergies: no peanut-/nut-containing products should be sold or served.

- The flight crew request that passengers in your immediate vicinity (a buffer area of at least one row of seats in front of and behind your seat) do not open or consume products containing peanuts/nuts (or the specific allergen). Since the risk of a severe allergic reaction from inhaling nut powder alone is very low, it does not appear necessary to inform all passengers unless such a reaction has occurred in the past.

4. If available, the person traveling should order allergen-free meals in good time. The airline cannot guarantee that these foods, or foods brought onto the aircraft by other passengers, are allergen-free. It is safer to bring your own food, whereby you need to check whether this is also permissible on connecting flights.

5. The airline should issue you with confirmation of the above-mentioned arrangements for presentation at check-in. If you do not inform the airline of your allergy until you are at the check-in, you may be denied boarding for safety reasons.

\section{On the day of travel}

1. Allow yourself plenty of time to go over your medications and special arrangements with security staff at the check-in. Although medications, including adrenaline autoinjectors, can be put through the scanner, they should always remain close to you and not stored, e.g., in the hand luggage compartment.

2. Ideally, those accompanying the allergy sufferer should clean arm rests, seats, and seat backs with wet wipes. Seat-back pockets should not be used, since food remnants that collect here are difficult to remove. You should also avoid using airline blankets or pillows.

3. You should request the flight crew to ask other passengers in the buffer area not to consume foods containing the relevant allergen. If no buffer area has been set up, you yourself can ask-but not demand-fellow passengers in your immediate vicinity to do so. You may need to ask to swap seats.

4. The flight crew should be immediately informed of any allergic reactions in order to request medical assistance and make other on-board emergency medications or oxygen available.

Funding Open Access funding enabled and organized by Projekt DEAL. 
Conflict of interest E. Rietschel is on the Mylan Advisory Board. J. Seidenberg, G. Stelljes, L. Lange and K. Blumchen declare that they have no competing interests.

Open Access This article is licensed under a Creative Commons Attribution 4.0 International License, which permits use, sharing, adaptation, distribution and reproduction in any medium or format, as long as you give appropriate credit to the original author(s) and the source, provide a link to the Creative Commons licence, and indicate if changes were made. The images or other third party material in this article are included in the article's Creative Commons licence, unless indicated otherwise in a credit line to the material. If material is not included in the article's Creative Commons licence and your intended use is not permitted by statutory regulation or exceeds the permitted use, you will need to obtain permission directly from the copyright holder. To view a copy of this licence, visit http://creativecommons.org/licenses/by/4.0/.

\section{References}

1. Sánchez-Borges M, Cardona V, Worm M, Lockey RF, Sheikh A, Greenberger PA, et al. In-flight allergic emergencies. World Allergy Organ J. 2017;10:15.

2. Casale TB, Lemanske RF Jr. In-flight medical emergencies. NEngl J Med. 2016;374:291-2.

3. Sicherer SH, Furlong TJ, DeSimone J, Sampson HA. Self-reported allergic reactions to peanut on commercial airliners. JAllergy Clin Immunol. 1999;104:186-9.

4. Comstock SS, DeMera R, Vega LC, Boren EJ, Deane S, Haapanen LAD, et al. Allergic reactions to peanuts, tree nuts, and seeds aboard commercial airliners. Ann Allergy Asthma Immunol. 2008;101:51-6.

5. BrockowK, Schallmayer S, Beyer K, Biedermann T, Fischer J, Gebert N, et al. Effects of a structured educational intervention on knowledge and emergency management in patients at risk for anaphylaxis. Allergy. 2015;70:227-35.

6. Edwards R. Ryanair bans passenger after girl suffers nut allergy reaction. 2014. www.telegraph.co.uk/travel/news/ ryanair/Ryanair-bans-passenger-after-girl-suffers-nutallergy-reaction/?WT.mc_id=tmg_share_em. Accessed 19 July 2020.

7. Oppermann M. Erdnüsse im Flugzeug. Skandalöses Verhalten bei Eurowings. 2018. www.daab.de/blog/2018/ 10/erdnuesse-im-flugzeug-skandaloeses-verhalten-beieurowings. Accessed 19 July 2020.

8. Stern. Passagierin muss wegen ihrer Nussallergie den Flug auf der Toilette verbringen. 2019. www.stern. $\mathrm{de} /$ reise/fernreisen/quantas--passagierin-muss-wegenihrer-nussallergie-flug-auf-der-toiletteverbringen-8621646.html. Accessed 27 May2020.

9. Greenhawt M, MacGillivray F, Batty G, Said M, Weiss C. International study of risk-mitigating factors and in-flight allergic reactions to peanut and tree nut. J Allergy Clin Immunol Pract. 2013;1:186-94.

10. IATA. Allergen sensitive passengers. 2016. www.iata.org/ contentassets/ccbdc54681c24574bebf2db2b18197a5/ allergen-sensitive-passenger.pdf. Accessed 19 July 2020.

11. Air Canada. Homepage. 2020. www.aircanada.com. Accessed 19July 2020.

12. EMA. Better training tools to support patients using adrenaline auto-injectors. 2015. https://www.ema.europa. $\mathrm{eu} / \mathrm{en} /$ documents/referral/adrenaline-auto-injectorsarticle-31-referral-better-training-tools-recommendedsupport-patients_en.pdf. Accessed 27 May2020.
13. Greenhawt M. Environmental exposure to peanut and the risk of an allergic reaction. Ann Allergy Asthma Immunol. 2018;120:476-81.

14. Jin JJ, Dorn JM, Yunginger J. Ara 2 is detectable on surfaces of commercial airplanes. J Allergy Clin Immunol Pract. 2019;7:659-61.

15. Jones RT, Stark DF, Sussmann GL, Yunginger JW. Recovery of peanut allergens from ventilation filters of commercial airliners. JAllergy Clin Immunol. 1996;97 (Suppl 1):423.

16. Perry TT, Conover-Walker MK, Pomés A, Chapman MD, Wood RA. Distribution of peanut allergen in the environment. J Allergy Clin Immunol. 2004;113:973-6.

17. Shaker M, Greenhawt M. Cost-effectiveness of stock epinephrine autoinjectors on commercial aircraft. J Allergy Clin Immunol Pract. 2019;7:2270-6.

18. Simons E, Sicherer SH, Simons FER. Timing the transfer of responsibilities for anaphylaxis recognition and use of an epinephrine auto-injector from adults to children and teenagers: pediatric allergists' perspective. Ann Allergy Asthma Immunol. 2012;108:321-5.

19. Ihr Europa. Anwendungsbereich der EU-Fluggastrechte. 2020. https://europa.eu/youreurope/citizens/travel/ passenger-rights/air/index_de.htm. Accessed 27 May 2020.

20. Aegean Airlines. Hilfe \& Kontakt. 2019. https://de. aegeanair.com/kontakt $/$ ?_ga $=2.252764361 .1183781837$. 1562141300-906438283.1562141300. Accessed 3 July 2019.

21. Aer Lingus. Dietary needs. 2019. www.aerlingus.com/ travel-information/special-assistance/dietary-needs/. Accessed 27 June 2019.

22. Aeroflot. Menüs an Bord. 2019. www.aeroflot.ru/ru-de/ information/onboard/dining. Accessed 7 July 2019.

23. Air Astana. Besondere Wünsche. 2019. https://airastana. com/ger/de-de/Air-Astana-Service/Besondere-Wuensch e/SondermahlzEite. Accessed 7 July 2019.

24. Air Baltic. Allergen info sheet. 2017. www.airbaltic. com/booking/meal/allergens/allergens_description_en. v3.pdf. Accessed 27 June 2019.

25. Air Baltic. Behinderte Passagiere. 2019. www.airbaltic. $\mathrm{com} / \mathrm{de} /$ behinderte-passagiere. Accessed 27 June 2019.

26. Air Canada. Unsere Barrierefreiheits-Services. 2019. www.aircanada.com/de/de/aco/home/plan/medicalmobility/policy-on-allergies.html. Accessed 3 July 2019.

27. AirChina. MedizinischeAusstattunganBord. 2019. https:// www.airchina.de/DE/DE/info/health-equipment/. Accessed 27 June 2019.

28. Air China. Spezialmenüs. 2019. www.airchina.de/DE/DE/ info/meals/special.html. Accessed 27 June 2019.

29. Air Dolomiti. Special assistance. 2019. www.airdolomiti. eu/special-assistance. Accessed 27 June 2019.

30. Air Europa. Fluggäste. 2019. https://www.aireuropa.com/ de/fluege/passagiere. Accessed 4 July 2019.

31. Air France. Do meals served on Air France flights contain peanuts? 2019. www.airfrance.com/LT/en/common/ faq/during-your-flight/do-meals- served-on-air-franceflights-contain-peanuts.htm. Accessed 3 July 2019.

32. Air France. Gesundheit und Wohlbefinden vor Reiseantritt 2019. www.airfrance.de/DE/de/common/guidevoyageur/ assistance/sante_avant_de_partir.htm. Accessed 3 July 2019.

33. Air France. Gesundheit und Wohlbefinden an Bord. 2019. www.airfrance.de/DE/de/common/guidevoyageur/ assistance/sante_a_bord.htm. Accessed 3 July2019.

34. Air France. Eingeschränkte Mobilität und andere Behinderungen an Bord. 2019. www.airfrance.de/DE/ de/common/guidevoyageur/assistance/pmr_a_bord_ airfrance.htm. Accessed 3 July 2019. 
35. Air India. Inflight experience. 2019. www.airindia.in/ inflight-experience.htm. Accessed 27 June 2019.

36. Air India. Disability assistance. 2019. www.airindia.in/ new-disability-assistance.htm. Accessed 3 July 2019.

37. Air Malta. Important information about food allergies. 2019. www.airmalta.com/information/services-by-airmalta/in-flight-catering/important-information-aboutfood-allergies. Accessed 8 July 2019.

38. Air Serbia. Medizinische Bedingungen und Bedingungen für Schwangere. 2019. www.airserbia.com/de-DE/ medizinische-bedingungen. Accessed 27 June 2019.

39. Air Serbia. Fitness to fly. 2019. https://www.airserbia.com/ data/documents/fitness-to-fly.pdf. Accessed 3 July 2019.

40. Alitalia. Spezielle Mahlzeiten. 2019. www.alitalia.com/ de_de/fly-alitalia/in-flight/special-meals.html. Accessed 4 July 2019.

41. ANA. Customers with allergies. 2019. www.ana.co.jp/ en/jp/serviceinfo/share/assist/support/allergy.html. Accessed 27 June 2019.

42. ANA. ANA policy regarding customers with peanut allergies. 2019. www.ana.co.jp/en/jp/international/ departure/inflight/spmeal/peanut.html. Accessed 27 June 2019

43. ANA. Q: Can I bring myinsulin syringes (injection needle) or EpiPen on board? 2019. www.ana.co.jp/en/jp/serviceinfo/ share/assist/faq/\#anchor009. Accessed 27June 2019.

44. ANA. Customers usually using autoinjectors (injection needles) and portable medical devices such as insulin pumps on board. 2019. www.ana.co.jp/en/jp/serviceinfo/share/ assist/support/medical/insulin.html. Accessed 27 June 2019.

45. American Airlines. Special meals and nut allergies. 2019. www.americanairlines.de/i18n/travel-info/experience/ dining/special-meals-and-nut-allergies.jsp. Accessed 4 July 2019.

46. Focus. Gilt ab 12. Dezember weltweit: Airline lässt Passagiere ihre Sitze säubern. 2018. www.focus. de/reisen/bei-us-airline-gilt-ab-12-dezember-weltweitairline-laesstpassagiere-ihre-sitze-saeubern_id_10011148. html. Accessed 4 July 2019.

47. Schlangenstein M. American Air to allow early boarding for those with nut allergies. 2018. https://www.bloomberg. com/news/articles/2018-11-06/american-air-to-allowearly-boarding-for-nut-allergy-sufferers. Accessed 4 July 2019.

48. Asiana Airlines. Passengers with medical assistance. 2019. https://flyasiana.com/C/US/EN/contents/medicalassistance-guide. Accessed 27 June 2019.

49. Asiana Airlines. Special meals. 2019. https://flyasiana. com/C/US/EN/contents/special-in-flight-meals. Accessed 27 June 2019.

50. Austrian. Spezialmenüs. 2019. www.austrian.com/Info/ Flightinformation/Special\%20Meals.aspx?sc_lang=de\& $\mathrm{cc}=\mathrm{AT}]$. Accessed 4 July 2019.

51. Austrian. GesundesReisen. 2019. www.austrian.com/Info/ Flying/MedicalInformation.aspx?sc_lang=de\&cc=AT. Accessed 4 July 2019.

52. Azores Airlines. Allergies. 2019. www.azoresairlines.pt/en/ information/special-services/allergies. Accessed 27 June 2019.

53. British Airways. Medical conditions and pregnancy. 2019. www.britishairways.com/de-de/information/ travel-assistance/medical-conditions-and-pregnancy. Accessed 4 July 2019.

54. British Airways. Food and drink. 2019. www.britishairways. $\mathrm{com} / \mathrm{en}$-gb/information/food-and-drink/food-allergies. Accessed 4 July2019.
55. Brussels Airlines. Was mache ich, wenn ich an einer Allergie leide? 2019. https://faq.brusselsairlines.com/l/de/ article/876p3xar37-was-mache-ich-wenn-ich-an-einerallergie-leide. Accessed 27 June 2019.

56. Brussels Airlines. Spezialmenüs. 2019. www. brusselsairlines.com/de-at/praktische-info/reise-info/ mein-ticket/spezialmenues/default.aspx. Accessed 27 June 2019.

57. Cathay Pacific. Physician's statement: peanut and tree nut allergy. 2019. https://www.cathaypacific.com/content/ $\mathrm{dam} / \mathrm{cx} /$ travel-information/special-assistance/medicalassistance/physician_statement_peanut_and_tree_nut_ allergies_201706.pdf. Accessed 27 June2019.

58. Cathay Pacific. Lebensmittelallergien. 2019. www. cathaypacific.com/cx/de_DE/travel-information/flyingwith-us/inflight-dining/allergies.html. Accessed 27 June 2019.

59. China Eastern Airlines. Besonderes Essen. 2019. https://de. ceair.com/newCMS/de/de/content/de_Header/header Bottom/service/Infomation/ticket/201904/t20190403_ 5603.html. Accessed 4 July 2019.

60. China Eastern Airlines. Special passenger application. 2019. https://us.ceair.com/en/special-serviceapplication- ossp.html?formtype=OSSP. Accessed 4 July 2019.

61. Croatia Airlines. Speisen und Getränke. 2019. www. croatiaairlines.com/de/Info-Service/An-Bord/Speisenund-Getranke. Accessed 1 July 2019.

62. Alpha Flight. Bistro. 2019. https://csawpcdnep01. azureedge.net/media/1878/bistro.pdf. Accessed 1 July 2019.

63. Delta. Ernährungsbedürfnisse und Allergien. 2019. https://de.delta.com/us/de/accessible-travel-services/ dietary-needs-and-allergies. Accessed 4 July 2019.

64. Delta. Richtlinie zu Erdnussallergien. 2019. https:// pro.delta.com/content/agency/erp/de/products-andservices/special-services/peanut-allergy-policy.html. Accessed 4 July 2019.

65. easyJet. Medizin, Gesundheitszustand und medizinische Ausrüstung. 2019. www.easyjet.com/de/hilfe/ boarding-und-fliegen/medikamente-und-medizinischeausrustung. Accessed 1 July 2019.

66. Egypt Air. Individuelle Menüwünsche. 2019. www.egyptair. $\mathrm{com} / \mathrm{de} / \mathrm{fly} /$ special-services/Seiten/special-meals.aspx. Accessed 1 July 2019.

67. Etihad. Medizinische Betreuung an Bord für Ihre Reise beantragen. 2019. www.etihad.com/de/experienceetihad/special-assistance/medical-information/. Accessed 1 July 2019.

68. Emirates. Individuelle Menüwünsche. 2019. www. emirates.com/de/german/before-you-fly/travel/dietaryrequirements.aspx. Accessed 1 July 2019.

69. Emirates. Besondere Hilfeleistungen. 2019. www.emirates. $\mathrm{com} / \mathrm{de} /$ german/before-you-fly/health/special-needs. aspx. Accessed 1 July 2019.

70. Finnair. Allergien. 2019. www.finnair.com/de/de/ information-services/before-the-flight/special-serviceshealth/allergies. Accessed 4 July 2019.

71. Finnair. FAQ. 2019. https://faq.finnair.com/AYKE FAQ_Search?ut=customer_site $\&$ country=de\&lang=de $\&$ siteLang=de. Accessed 4 July 2019.

72. Finnair. GesundheitlicheBeschwerden. 2019. www.finnair. $\mathrm{com} / \mathrm{de} / \mathrm{de} /$ information-services/before-the-flight/ special-services-health/medical-conditions. Accessed 4 July 2019.

73. Flybe. Menu. 2019. www.flybe.com/menu. Accessed 4 July 2019. Flybe UKbusiness has ceased trading March 2020 . 
74. Flybe. Passenger assistance. 2019. https://flybe.custhelp. $\mathrm{com} / \mathrm{app} /$ answers/detail/a_id/1347\#gs=eyJndWlkZUlEIjo 5NywicXVlc3Rpb25JRCI6MSwicmVzcG9uc2VJRCI6NywiZ3 VpZGVTZXNaW9uIjoiSnFSYmdFaW8iLC JzZXNzW9uSUQiOiJjOVpLZkVpbyJ9. Accessed 4 July 2019. Flybe UKbusiness has ceased trading March 2020.

75. Flybe. Allergy. 2019. https://flybe.custhelp.com/app/ answers/detail/a_id/118/kw/allergy. Accessed 4 July 2019. Flybe UKbusiness has ceased trading March 2020.

76. Flybe. Allergy. 2019. https://flybe.custhelp.com/app/ answers/detail/a_id $/ 739 / \mathrm{kw} /$ allergy/related $/ 1$. Accessed 4 July 2019. Flybe UK business has ceased trading March 2020.

77. Iberia. Gastronomy. 2019. http://help.iberia.com/ frequently_asked_question/gastronomy. Accessed 7 July 2019.

78. Icelandair. Reisen mit gesundheitlichen Einschränkungen. 2019. www.icelandair.com/de-de/hilfe/besonderebetreuung/reisen-mit-gesundheitlicheneinschraenkungen. Accessed 4 July 2019.

79. Japan Airlines. Customers with food allergies. 2019. www. jal.co.jp/en/jalpri/support/allergy.html. Accessed 1 July 2019.

80. Japan Airlines. Minimal allergen meals. 2019. www.jal. co.jp/en/inter/service/meal/special/menu/allergen. Accessed 1 July 2019.

81. KLM. Nuss- oder Erdnussallergie und sonstige Lebensmittelallergien. 2019. www.klm.com/travel/de_de/ prepare_for_travel/on_board/dining_on_board/special_ meals.htm\#p6. Accessed 1 July 2019.

82. KLM. Besondere Betreuung während Ihrer Reise. 2019. www.klm.com/travel/de_de/prepare_for_travel/travel planning/special_assistance/klm_cares.htm. Accessed 1 July 2019.

83. Korean Air. Hinweise für Fluggäste mit Erdnussallergie. 2019. https://www.koreanair.com/global/ $\mathrm{de} /$ traveling/classes-of-service/in-flight-special-meals/ peanut-allergy/. Accessed 1 July 2019.

84. Kuwait Airways. Onboard services. 2019. www. kuwaitairways.com/en/is/onboard-service. Accessed 1 July 2019.

85. Laudamotion. Ichleide an einer Nussallergie. 2019. https:// www.laudamotion.com/la/de/nutzliche-infos/servicecenter/haufige-fragen/Besondere-hilfeleistungen/Wer den-in-den-mahlzeiten-an-bord-von-Ryanair-flugen-erd nusse-oder-andere-nusse-verarbeitet-Ich-leide-an-einernussallergie-und-mochte-wissen-ob-es-bei-Ryanair-einerichtlinie-zur-verwendung-von-nussen-in-mahlzeitengibt. Accessed 20. October 2020.

86. Lot. Sondermahlzeiten. 2020. https://www.lot.com/de/ $\mathrm{de} / \mathrm{special}-\mathrm{meal}$. Accessed 20 October 2020

87. LOT. Passagiere mit ärztlicher Bescheinigung. 2019. www. lot.com/de/de/passagiere-mit-arztlicher-bescheinigung. Accessed 1 July 2019

88. Lufthansa. Allergien. 2019. www.lufthansa.com/se/de/ gesund-reisen\#allergies. Accessed 1 July 2019.

89. Norwegian Air. Krankheiten an Bord. 2019. www. norwegian.com/de/reiseinfo/besondere-hilfeleistung/ erkrankungen. Accessed 1 July 2019.

90. Oman Air. General conditions of carriage for Oman Air. 2019. https://sindbad.omanair.com/en/legal/conditionscarriage. Accessed 8 July 2019.

91. Pegasus. Passagiere mit Eingeschränkter Mobilität und Gesundheit. 2019. https://www.flypgs.com/de/nutzlicheinfos/andere-informationen/passagiere-mit-eingeschr \%C3\%A4nkter-mobilit\%C3\%A4t-und-gesundheit. Accessed 1 July 2019.
92. Qantas. Taking medical equipment and medications onboard. 2019. www.qantas.com/travel/airlines/medicalequipment-and-medications/global/en. Accessed 8 July 2019.

93. Qantas. Fitness to fly. 2019. www.qantas.com/travel/ airlines/medical-assistance/global/en. Accessed 8 July 2019.

94. Qatar Airways. What is the allergy policy on Qatar Airways? 2019. https://qatarairways.zendesk.com/hc/en-us/ articles/206466748-Allergy-Policy. Accessed 1 July 2019.

95. Qatar Airways. Special meals. 2019. www.qatarairways. $\mathrm{com} / \mathrm{en} / \mathrm{services}$-special/special-meals.html. Accessed 1 July 2019.

96. Royal Jordanian. Traveling with special needs - ADA 2019. www.rj.com/en/info-and-tips/special-services/ passenger-with-special-needs-ada. Accessed 4 July 2019.

97. Ryanair. Nussallergie. 2020. https://www.ryanair.com/ at/de/nutzliche-infos/service-center/haufige-fragen/ Besondere-hilfeleistungen/Werden-in-den-mahlzeitenan-bord-von-Ryanair-flugen-erdnusse-oder-andere-nus se-verarbeitet-Ich-leide-an-einer-nussallergie-und-moch te-wissen-ob-es-bei-Ryanair-eine-richtlinie-zur-verwendung-von-nussen-in-mahlzeiten-gibt. Accessed 20 October 2020.

98. Singapore Airlines. Passagiere mit Nussallergie. 2019. www.singaporeair.com/de_DE/de/travel-info/requests/ passengers-with-nut-allergy/. Accessed 2 July 2019.

99. SAS. Spezielle Mahlzeiten. 2019. www.flysas.com/ de-de/reiseinformationen/besondere-reisebedurfnisse/ spezielle-mahlzeiten/. Accessed 4 July 2019.

100. SAS. Kann ich meine eigenen Lebensmittel mit an Bord nehmen? 2019. www.flysas.com/de-de/hilfeund-kontakt/faq/travel-info/kann-ich-meine- eigenenlebensmittel-mit-an-bord-nehmen. Accessed 4 July 2019.

101. South African Airways. Menüs an Bord. 2019. www.flysaa. com/de_DE/manage-fly/during-the-flight/menus. Accessed 2 July 2019.

102. South African Airways. Nussallergie. 2019. http:// flysaatrade.com/de/a-z/essengetrnke-1\#nussallergie. Accessed 2 July 2019.

103. Sundair. Bord-Bistro. 2019. https://www.sundair.com/ downloads/Sundair-Bord-Bistro_and_Travel-Value.pdf. Assessed 2 July 2019

104. Sunexpress. Extras und mehr. 2020. https://www. sunexpress.com/de/buchen/extras-und-mehr/an-bord/. Accessed 20 October 2020.

105. Sunexpress. SunCafe. 2020. https://cdn.sunexpress. com/wp-content/uploads/sites/3/2020/07/09173038/ SunCafe.pdf. Accessed 20 October 2020.

106. SunExpress. Wir unterstützen Passagiere mit besonderen Bedürfnissen. 2019. www.sunexpress.com/ de/information/passagierinformationen/fluggaeste-mitbehinderung. Accessed 2 July 2019.

107. Swiss International Air Lines. Erdnuss-Allergie. 2019. www. swiss.com/corporate/medicalservices/de/gesundheit/ fliegen-mit- einem-gebrechen/flying-with-apeanut-allergy. Accessed 2 July 2019.

108. Swiss International Air Lines. Gesundheitund Reisen. 2019. www.swiss.com/de/de/vorbereiten/spezielle-betreuung/ gesundheit-und-reisen. Accessed 2 July 2019.

109. Swiss International Air Lines. Spezialmenüs. 2019. www.swiss.com/de/DE/fliegen/an-bord/verpflegung/ spezialmenues. Accessed 2 July 2019.

110. TAP. Meals on board. 2019. www.flytap.com/en-il/onboard $/$ meals- on-board? accordionid=4. Accessed 2 July 2019. 
111. ThaiAirways. GesundheitundWohlbefinden anBord. 2019. www.thaiairways.com/de_CH/plan/travel_information/ health_and_well_being_onboard.page. Accessed 2 July 2019.

112. Thai Airways. Gesundheit und Wohlbefinden an Bord. 2019. www.thaiairways.com/de_DE/plan/info_service/ gesundheit_und_wohlbefinden_an_bord.page. Accessed 2 July 2019.

113. TUI. TUI fly Café Karte. 2019. www.tuifly.com/downloads/ TUI_fly_Cafe_Karte_01_2019_20190423.pdf. Accessed 8 July 2019.

114. Turkish Airlines. Wird während des Fluges Erste Hilfe geleistet? 2015. www.turkishairlines.com/deint/any-questions/can-i-get-first-aid-treatment-duringthe-flight/index.html. Accessed 2 July 2019.

115. Turkish Airlines. Special meal service. 2019. www. turkishairlines.com/de-int/any-questions/special-meals/ Accessed 2 July 2019.

116. United Airlines. Kunden mit Nahrungsmittelallergie. 2019. www.united.com/ual/de/de/fly/travel/specialneeds/food-allergies.html. Accessed 2 July 2019.
117. United Airlines. Hilfestellung während des Fluges. 2019. www.united.com/ual/de/de/fly/travel/specialneeds/disabilities/inflight-assistance.html. Accessed 2 July 2019.

118. United Airlines. Nützliche Informationen für Kunden mit Behinderungen. 2019. www.united.com/ual/de/de/ fly/travel/special-needs/disabilities/useful-info.html. Accessed 2 July 2019.

119. Vueling. Homepage. 2019. www.vueling.com/de. Accessed 2 July 2019.

120. Wizzair. Besondere Hilfeleistungen bei Flugstörungen und Passagieren mit Behinderungen. 2019. wizzair.com/de-de/informationen-und-serviceleistungen/ reiseinformationen/besondere-hilfeleistungen. Accessed 8July 2019.

121. Anaphylaxis Campaign. Airline allergy policies. 2019. www. anaphylaxis.org.uk/living-with-anaphylaxis/travelling/ airline-allergy-policies. Accessed 12 July 2019. 